\title{
Alteración de la Conciencia Secundario al Mal Control Metabólico, Reportado en el Servicio de Emergencia del Hospital General Iess Riobamba
}

\author{
Alteration of Secondary Consciousness to Poor Metabolic Control, Reported \\ in the Emergency Service of the Iess Riobamba General Hospital
}

Adrián Paul Llerena Asadobay ${ }^{1}$, Paola Belén Salazar Montero², Evelyn Carolina Villarroel Ponce ${ }^{3}$, Sandy Yomary Riofrío García ${ }^{4} \&$ Nelson Mauricio Moreno Moreira ${ }^{5}$

\begin{abstract}
. https://doi.org/10.32/cienciadigital.v3i1.947

Diabetes Mellitus (DM) comprises a group of metabolic disorders that share the common hyperglycemia phenotype. Classifying into: DM type 1 , it is characterized by early insulin deficiency (2). Type $2 \mathrm{DM}$ is characterized by insulin resistance and excessive production of glucose in the liver. The physical examination, laboratory tests such as blood glucose, elementary and microscopic urine, arterial blood gases (1), are the most effective methods to determine early acute complications such as: ketoacidosis, hyperosmolar non-ketotic state and hypoglycemia. Objective: to present the clinical elements and laboratory tests that allow to reach the diagnosis of this disease. Clinical case: We present a male patient of 41 years of age, who attended the IESS General Hospital of Riobamba in which a specific case of Alteration of consciousness secondary to poor metabolic control was presented.

Conclusions: the alteration of consciousness secondary to poor metabolic control, greatly affects the biopsychosocial well-being of patients who suffer from it, being

\footnotetext{
${ }^{1}$ Médico General. Estudiante de Postgrado en Dirección en Gerencia Hospitalaria -Universidad Internacional de la Rioja. Riobamba - Ecuador. adrianchuspr@hotmail.com @intitutos.gob.ec

${ }^{2}$ Master en Prevención de Riesgos Laborales. Médico General. Docente del Instituto Tecnológico Superior Riobamba. Riobamba-Ecuador. paosalazarm88@hotmail.com psalazar@intitutos.gob.ec

3 Médico General. Docente del Instituto Tecnológico Superior Riobamba. Riobamba - Ecuador. joflim98@hotmail.com evillarroel@intitutos.gob.ec

${ }^{4}$ Médico General. Docente del Instituto Tecnológico Superior Riobamba. Riobamba - Ecuador. yomary_2009@hotmail.com sriofrio@intitutos.gob.ec

${ }^{5}$ Médico General. Docente del Instituto Tecnológico Superior Riobamba. Riobamba - Ecuador. morenomauricio@live.com.gmoreno@intitutos.gob.ec
} 
important to master all clinical aspects and laboratory tests that make us suspect their diagnosis to minimize the complications that arise from it. they can be derived.

Keywords: Diabetes Mellitus; Alteration of the conscience; diabetic Cetoacidosis; the State Hiperosmolar not cetosico; Hypoglycemia.

\section{Resumen.}

La Diabetes Mellitus (DM) comprende un grupo de trastornos metabólicos que comparten el fenotipo común de la hiperglucemia. Clasificándose en: DM tipo 1, se caracteriza por el déficit temprano de insulina (2). La DM tipo 2 se caracteriza por resistencia a la insulina y producción excesiva de glucosa en el hígado. La realización del examen físico, exámenes de laboratorio como glucosa en sangre, elemental y microscópico de orina, gasometría arterial (1), son los métodos más efectivos para determinar a tiempo las complicaciones agudas como: cetoacidosis, estado hiperosmolar no cetosico e hipoglicemia. Objetivo: dar a conocer los elementos clínicos y exámenes de laboratorio que permitan llegar al diagnóstico de ésta enfermedad. Caso clínico: Se presenta un paciente masculino de 41 años de edad, que acude al Hospital General IESS de Riobamba en el que se presentó un caso específico de Alteración de la conciencia secundario al mal control metabólico.

Conclusiones: la alteración de la conciencia secundaria al mal control metabólico, afecta considerablemente el bienestar biopsicosocial de los pacientes que la padecen, siendo importante dominar todos los aspectos clínicos y exámenes de laboratorio que nos hacen sospechar de su diagnóstico para minimizar las complicaciones que de ella se pueden derivar.

Palabras clave: Diabetes Mellitus; Alteración de la conciencia; Cetoacidosis diabética; Estado Hiperosmolar no cetosico; Hipoglicemia.

\section{Introducción.}

Los trastornos metabólicos que se caracteriza por hiperglucemia es la diabetes mellitus (DM), debida a defectos en la secreción de la insulina. Los procesos fisiopatogénicos involucrados en su aparición varían desde la destrucción autoinmunitaria de las células $\beta$ del páncreas hasta alteraciones en la resistencia de la insulina.(1)

Se clasifica en categorías clínicas: - DM tipo 1 (DM1), que se divide en: - Autoinmune (DM1 A). - Idiopática (DM1B). DM tipo 2 (DM2). • DM gestacional. (1) 
La DM1 afecta al 5-10 \% de la población diabética. Se caracteriza por una destrucción de las células $\beta$ del páncreas, que da lugar a un déficit absoluto de insulina, suele deberse a un mecanismo autoinmune. (1) La DM2 supone el 85-95 \% de los casos de DM, y se caracteriza por una resistencia a la insulina combinada con un déficit progresivo de producción de esta. (1)

La Organización Mundial de la Salud (OMS), estima que hay más de 347 millones de personas con diabetes en el 2016. Más del $80 \%$ de las muertes por diabetes se registran en países de ingresos bajos y se prevé que las muertes por diabetes se multiplicarán por 2 entre 2017 y 2030. (2) La Federación Internacional de Diabetes declara que en el Ecuador la prevalencia de la enfermedad en adultos entre 20 a 79 años es del $8.5 \%$.(3)

Según la Guía de Practica Clínica de la Diabetes Mellitus tipo 2 del 2017 por parte del Ministerio de Salud del Ecuador se estima que la incidencia de la diabetes mellitus en la provincia de Chimborazo es del $7.3 \%$ teniendo una edad de referencia de 30 a 60 años.(2)

En cuanto al sexo existe un predominio moderado de mujeres, con una relación 1,2:1 aproximadamente respecto a los varones. La incidencia (casos nuevos por año por cada 100.000 habitantes) de DM se estima en 200 para el grupo de edad de 40-49 años, 350 de 5059,600 de 60-69 y alrededor de 800 después de los 70 años. La incidencia y la prevalencia de la DMNID dependen en gran medida del índice de masa corporal y de la actividad física.(5)

\section{Marco Teórico}

Los principales síntomas de la diabetes mellitus son la poliuria, polidipsia, pérdida de peso, fatiga, visión borrosa, infecciones superficiales frecuentes y mala cicatrización de las heridas. Para diagnosticar es necesario una glucosa en ayunas, prueba de tolerancia oral a la glucosa con 75 gramos de glucosa anhidra y glucemia al azar en pacientes con síntomas de síndrome diabético agudo. - Necesidad de estandarización de la técnica para el uso de hemoglobina glicosilada A1c (HbA1c) para diagnóstico.(6)

El diagnóstico de DM2 se realizará en pacientes que cumplan al menos uno de los siguientes criterios: 1. Glucemia de ayuno medida en plasma venoso que sea igual o mayor a $126 \mathrm{mg} / \mathrm{dL}$ ( $7.0 \mathrm{mmol} / \mathrm{L}$ ), confirmada con una segunda prueba en diferentes días. (Ayuno se define como un período sin ingesta calórica de por lo menos ocho horas). 2. Glucemia medida en plasma venoso que sea igual o mayor a $200 \mathrm{mg} / \mathrm{dL}(11.1 \mathrm{mmol} / \mathrm{L})$ dos horas después de una carga de $75 \mathrm{~g}$ de glucosa anhidra durante una prueba de tolerancia oral a la glucosa (PTOG). 3. Pacientes con polifagia, poliuria, polidipsia y pérdida inexplicable de peso, más una glucemia al azar medida en plasma venoso que sea igual o mayor a $200 \mathrm{mg} / \mathrm{dL}(11.1 \mathrm{mmol} / \mathrm{L})$. 4. Una 
HbA1c mayor o igual a $6.5 \%(48 \mathrm{mmol} / \mathrm{mol})$, empleando una metodología estandarizada y trazable al estándar.

Como tratamiento se recomienda asociar metformina a cambios de hábitos de vida, siempre y cuando no haya contraindicaciones a esta medicación, el paciente no se encuentre en cetosis o con baja de peso significativa. Se recomienda comenzar con dosis bajas de metformina (500mg), llevando titulaciones progresivas hasta una dosis máxima de $2550 \mathrm{mg}$ en controles periódicos, al menos de manera trimestral con el control de HbA1c. Nota: en el adulto mayor la dosis máxima recomendada es de $1700 \mathrm{mg}$ diario. En pacientes con intolerancia gastrointestinal comprobada a metformina, pese a inicios a dosis bajas y aumento progresivo de dosis, se recomienda intentar con metformina de acción prolongada ya que ha demostrado menor frecuencia de este evento adverso, manteniendo su eficacia clínica. La elección de este antidiabético oral se debe a que ha demostrado disminución de la mortalidad cardiovascular y menor cantidad de efectos adversos en comparación con otros antidiabéticos orales. Se debe ajustar la dosis de metformina en pacientes con aclaramiento de creatinina entre 30 y $60 \mathrm{~mL} / \mathrm{min} / 1.73 \mathrm{~m} 2$. No se debe prescribir metformina a los pacientes que presenten las siguientes contraindicaciones: 1. Hipersensibilidad (diarrea, nausea, vómito, cefalea, etc) 2. Durante la cetoacidosis diabética y el estado hiperosmolar hiperglucémico. 3. Insuficiencia renal (aclaramiento de creatinina, Clcr menor a $30 \mathrm{ml} / \mathrm{min} / 1.73 \mathrm{~m} 2$ ). 4. Insuficiencia cardíaca con fracción de eyección.(4)

La cetoacidosis diabética (CAD) era la primera causa de muerte por diabetes con el $40 \%$. Constituye una causa importante de morbilidad en pacientes diabéticos mal tratados. Se desarrolla en varias fases y se caracteriza inicialmente por una producción aumentada de cuerpos cetónicos, con elevadas concentraciones plasmáticas de los ácidos acetoacético e hidroxibutírico. La causa última es la insulinopenia grave. El déficit de insulina es responsable del aumento de la gluconeogénesis y de la glucogenólisis, El grave déficit de insulina se produce como consecuencia de que ésta no ha sido administrada en los días anteriores o lo ha sido en dosis insuficientes o bien porque las necesidades de insulina han aumentado de forma importante. (7)

Hay dos signos clínicos que llaman la atención; la deshidratación y la respiración de Kussmaul que es una taquipnea típica que se presenta cuando el pH es inferior a 7,20 o 7,10. Se acompaña del clásico aliento a manzanas y, cuando es muy acusada, puede llegar a provocar intensa fatiga. (8) La hipotermia es frecuente, como consecuencia de la pérdida de calor por la vasodilatación periférica cutánea inducida por la acidosis. Un individuo deshidratado, con respiración profunda y rápida, que presenta glucosuria y cetonuria máximas en orina y cuerpos cetónicos en plasma padece con seguridad una CAD.(5)

La insulina se utiliza en forma simple y vía intravenosa. Dosis usual inicial es de 6-10 U/h en perfusión continua $(0,1 \mathrm{U} / \mathrm{kg} / \mathrm{h})$, diluyendo en una solución salina al $0,9 \%$ proporción 
aproximada de $1 \mathrm{U} / \mathrm{mL}$, se puede añadir seroalbúmina o un expansor plasmático sintético para prevenir adherencia de la insulina a las paredes del sistema de perfusión.(5)

Coma hiperglucémico hiperosmolar no cetósico (CHHNC), se trata de individuos de edad avanzada que sufren una deshidratación acusada y tienen cifras de glucemia muy elevadas (superiores a $600 \mathrm{mg} / \mathrm{dl}$ ) y, a menudo, hipernatremia. El CHHNC se define metabólicamente por la ausencia de acidosis y una osmolalidad plasmática efectiva (OPE) superior a 320 mosm/L o una osmolalidad plasmática total (OPT) superior a $340 \mathrm{mosm} / \mathrm{L}$. La hiperglucemia sin acidosis se puede producir por varios mecanismos: a) presencia de secreción residual de insulina endógena con insulinemia periférica, que es capaz de impedir la lipólisis pero no la hiperglucemia; b) concentraciones de insulina adecuadas en la circulación hepática pero insuficientes en la periférica, con lo que la lipólisis aumentada deriva hacia gluconeogénesis y no hacia cetogénesis, y c) la deshidratación hiperosmolar que impiden la lipólisis. (9)

El pronóstico del CHHNC es mucho más sombrío y su mortalidad oscila entre el 40 y el $70 \%$ en algunas series. Los factores desencadenantes del CHHNC son similares a los mencionados en la CAD, con la diferencia de que la etiología infecciosa es más frecuente, pueden intervenir algunos fármacos como glucocorticoides, tiazídicos, bloqueadores beta, cimetidina, clorpromazina, difenilhidantoína, inmunodepresores, diazóxido o furosemida, y a menudo se desconoce la existencia de una diabetes previa.(9)

Por otra parte, el inicio de las manifestaciones clínicas suele ser aún más insidioso, con incremento gradual de la obnubilación, alucinaciones, hemianopsias, afasias, nistagmo, alteraciones sensoriales, convulsiones y hemiplejías hasta llegar al coma. La afectación de la conciencia es mucho más grave que en la CAD y, a diferencia de ésta, más del $80 \%$ de los pacientes están realmente en coma y el resto presenta grados variables de obnubilación. La deshidratación es aún más importante que en la CAD y se halla agravada por la disminución de la sensibilidad de los centros de la sed que presentan a veces las personas de edad avanzada.(7)

El tratamiento de estos pacientes debe perseguir fundamentalmente los siguientes objetivos: corregir la hipovolemia y la hiperosmolalidad mediante hidratación, normalizar la glucemia con insulina, corregir los déficit electrolíticos concomitantes si existen, investigar y tratar el factor desencadenante y, por último, prevenir en lo posible las complicaciones. La evolución de los pacientes de $\mathrm{CHHNC}$ es en general desfavorable. El pronóstico guarda relación sobre todo con la edad, la profundidad del coma y la hiperosmolalidad. (1)

Debido a la escasa presencia de casos de Alteración de la conciencia secundario a un mal control metabólico en el Ecuador, la investigación se enfoca en profundizar el tema en cuanto a causas, clínica, complicaciones y tratamiento de pacientes con Diabetes Mellitus, basados 
en un caso en específico y con el cual obtendremos datos e información relevante para un mayor conocimiento de esta enfermedad.

\section{Hipoglucemia}

La hipoglucemia es un síndrome clínico de etiología diversa, en el que los niveles bajos de glucosa sérica pueden conducir al final a neuroglucopenia. (8)

La clasificación tradicional de la hipoglucemia en dos grandes grupos, de ayuno o postabsortiva y posprandial o reactiva, ha sido sustituida actualmente por una categorización clínica en función de la existencia o no de DM, y en ausencia de DM la aparición de hipoglucemia se contempla en dos marcos diferentes: en circunstancias de enfermedad o en situación aparente de salud. (6)

Existen varios criterios que tienen en cuenta este nuevo enfoque y que pueden servir de ayuda en el reconocimiento de la causa y en el planteamiento del diagnóstico diferencial: 1) la hipoglucemia más frecuente, con gran diferencia sobre las otras etiologías, es la que aparece en la mayoría de los pacientes con DM tipo 1, y también en muchos con tipo 2, en tratamiento intensivo con insulina, con sulfonilureas o ambos; 2) la hipoglucemia considerada de ayuno puede dar lugar a sintomatología de aparición posprandial, y, al contrario, la hipoglucemia posprandial puede cursar con clínica en situación de ayuno; 3) en sujetos aparentemente sanos, la ingesta equivocada o deliberada de sustancias o fármacos, incluidos la insulina y los medicamentos inductores de su secreción, constituye la causa más frecuente de hipoglucemia; 4) en los pacientes hospitalizados con enfermedades graves, la acción de los fármacos, incluidos la insulina o los agentes hipoglucemiantes, en el marco de una mal nutrición, fallos orgánicos o infecciones graves, es responsable del cuadro hipoglucémico, y 5) la presencia de sintomatología hipoglucémica posprandial, sin que se demuestre un descenso en la concentración plasmática de glucosa, como se demuestra la mayoría de las veces, se considera actualmente un trastorno funcional en el que los síntomas no son debidos a hipoglucemia y cuyo reconocimiento se engloba actualmente bajo el término de seudohipoglucemia. (6)

Las guías de práctica clínica de la Sociedad Americana de Endocrinología (ES, por sus siglas en inglés, Endocrine Society) indican que se realice la evaluación y manejo de la hipoglucemia sólo en pacientes que presenten la tríada descrita desde 1938 por Whipple y que consiste en: 1. Síntomas, signos o ambos sugestivos de hipoglucemia, 2. Una concentración de glucosa plasmática baja, y 3. Resolución de los síntomas o signos después de que la concentración de glucosa plasmática aumenta.(9)

Los Síntomas adrenérgicos de la hipoglicemia son palpitaciones, ansiedad, temblor, palidez, sensación de hambre. Síntomas colinérgicos: diaforesis, náuseas. Síntomas 
neuroglucopénicos: cefalea, debilidad, alteración del comportamiento, dificultad para concentrarse, ataxia, disminución del nivel de conciencia, focalidad neurológica, convulsiones. Cabe destacar que hasta el $40 \%$ de las personas diabéticas en tratamiento con insulina tienen episodios de hipoglucemia nocturna; las respuestas adrenérgicas están disminuidas durante el sueño profundo, lo que contribuye a no despertarse durante el episodio. Síntomas orientadores de hipoglucemia nocturna son: pesadillas, diaforesis, cefalea y astenia al levantarse, y enuresis nocturna.(10)

El punto de corte para definir hipoglucemia ha sido definido arbitrariamente por algunos autores. Carroll en su revisión propone como criterio de hipoglucemia $50 \mathrm{mg} / \mathrm{dL}$ con síntomas y $40 \mathrm{mg} / \mathrm{dL}$ en ausencia de síntomas. En las guías de la Sociedad Americana de Endocrinología, el punto de corte de hipoglucemia se ha establecido considerando que los síntomas se desarrollan en personas sanas cuando la concentración de glucosa plasmática está alrededor de $55 \mathrm{mg} / \mathrm{dL} .(9)$

El tratamiento de los trastornos hipoglucémicos engloba dos componentes distintos: 1) alivio de los síntomas neuroglucopénicos al aumentar el bajo nivel de la glucosa sérica hasta el intervalo normal y 2) corrección de la causa subyacente de hipoglucemia.(8)

\section{Metodologia.}

Este estudio es descriptivo situándose sobre una base de conocimientos más sólida que los exploratorios. En estos casos el problema científico ha alcanzado cierto nivel de claridad, pero aún se necesita información para poder llegar a establecer caminos que conduzcan al esclarecimiento de relaciones causales.

En el área de la investigación clínica son habituales los estudios que describen casos médicos puntuales y de los diferentes cuadros clínicos y los que examinan la asociación de características clínicas con signos radiológicos, histopatológicos, bioquímicos, etc. Para lo cual en el presente trabajo se ha descrito un conjunto de enfermedades presentadas posterior al examen médico en la unidad de emergencias de un Hospital de Segundo Nivel, determinando de esta manera la frecuencia de su presentación y siendo además analítico ya que consiste en la desmembración de un todo, descomponiéndolo en sus partes o elementos para observar las causas, la naturaleza y los efectos, en el contexto de la retrospectiva y el análisis clínico. Describiendo entonces a esta investigación como analítica, ya que se realiza observación mediante el examen médico, diagnóstico, tratamiento y complicaciones presentados en el caso en estudio.

\section{Resultados}


Se trata de un paciente masculino de 41 años de edad, nacido en Riobamba y residente en Riobamba, de raza mestiza, religión católica, estado civil casado, secundaria completa, su ocupación es comerciante, diestro, tipo de sangre y factor O Rh positivo.

Posee diabetes mellitus tipo 2 diagnosticado hace 2 años, en tratamiento con insulina NPH 20 UI subcutáneo en la mañana diariamente, además hipotiroidismo hace 8 años en tratamiento con Levotiroxina $50 \mathrm{mg}$ vía oral diariamente.

Antecedentes familiares manifiesta que la madre y hermano posee diabetes mellitus tipo 2.

El motivo por lo que la paciente acude al servicio de emergencia de esta casa de salud fue por la alteración de conciencia.

Esposa del paciente refiere que hace 2 horas aproximadamente, teniendo como causa aparente danzar, presenta alteración de la conciencia, por tiempo indeterminado, por lo que familiares intentan despertar a paciente sin respuesta favorable, posteriormente acude a esta casa de salud, en el servicio de emergencia recobra la conciencia espontáneamente con deseos de ingerir abundante agua, además de nauseas que llegan al vomito por una ocasión de contenido alimentario, se realiza test de glucosa capilar evidenciando valores por encima de $600 \mathrm{mg} / \mathrm{dl}$, por lo que se decide su ingreso.

Al examen físico el paciente se encuentra somnoliento, orientado en el tiempo, espacio y persona, con mucosas orales secas. Se le tomó los signos vitales, de los cuales los resultados muestran que la TA es de 130/80 mmHg. FC de 114 latidos por minuto. La FR es de 22 por minuto. La saturación de oxigeno es de $91 \%$. Su temperatura axilar es de $37.2^{\circ} \mathrm{C}$. Su peso es de 70 kilogramos. Su talla es de 165 centímetros. IMC 25.7 por lo que presenta Sobrepeso Grado I.

Cabeza normo cefálico, no presenta masas. Ojos se muestra escleras anictéricas, pupilas isocóricas normoreactivas a la luz y acomodación. Boca se muestra mucosas orales secas sin signos de lesión patológica. Lengua saburral con movimiento normal. Dientes presenta piezas dentales completas las cuales están en buen estado. Orofaringe no congestiva, no purulenta. Nariz con implantación normal, no desviación de tabique nasal, fosas nasales permeables. Oídos permeables conducto auditivo externo con cerumen en poca cantidad. Cuello simétrico sin ingurgitación yugular, no adenopatías. Tiroides no visible ni palpable (OA). Tórax simétrico, expansible, no alteración a la exploración. Pulmones presencia de murmullo vesicular conservado, no se ausculta ruidos sobre añadidos. Corazón ruidos cardiacos rítmicos, normo fonéticos en dos tiempos, no soplos. Abdomen suave, depresible levemente doloroso a la palpación a nivel de puntos renoureterales superiores y medios bilaterales, ruidos hidroaéreos presentes

Región lumbar: no se palpan masas, puño percusión positivo bilateral en leve intensidad 6/10. 
Región inguinogenital: genitales externos aparentemente normal, se evidencia sonda vesical permeable con orina concentrada.

Extremidades, miembros superiores e inferiores simétricos con buena movilidad, tono y fuerza conservada, no se evidencia edema. El resto de la exploración física es normal.

\begin{tabular}{llll}
\hline Signos y síntomas & Examen físico hallazgos & & \\
\hline Alteración de la conciencia. & En los signos vitales presenta taquicardia, \\
Polidipsia & taquipnea, febrícula. & & \\
Nauseas que llegan a vomito de & Mucosas orales secas. & \\
contenido alimentario por una & Dolor localizado en puntos renoureterales \\
ocasión. & superiores y medios bilaterales. & \\
\hline
\end{tabular}

Tabla $\mathbf{N}^{\circ}$ 1: Manifestaciones Clínicas de descompensación metabólica del paciente

Fuente: Hospital General IESS Riobamba, 01 de marzo del 2017.

\begin{tabular}{|c|c|c|}
\hline Paciente & Factores de riesgo & $\begin{array}{l}\text { Cambios fisiológicos en } \\
\text { pacientes con diabetes mellitus } \\
\text { tipo } 2\end{array}$ \\
\hline & $\begin{array}{l}\text { Antecedente personal de } \\
\text { diabetes mellitus. } \\
\text { Mal manejo terapéutico por } \\
\text { parte del paciente. } \\
\text { Sobrepeso Grado I. }\end{array}$ & $\begin{array}{l}\text { Cambios en el sistema } \\
\text { circulatorio. } \\
\text { Cambios Físicos del paciente. } \\
\text { Cambios Nutricionales }\end{array}$ \\
\hline
\end{tabular}

Tabla $\mathrm{N}^{\circ}$ 2: Factores de riesgo.

Fuente: Historia Clínica del Paciente - Hospital General IESS Riobamba. 1 de marzo del 2017. EXÁMENES COMPLEMENTARIOS

\begin{tabular}{ll}
\hline Tipificación & \\
\hline Grupo y factor: & O Rh positivo. \\
Biometría hemática: & \\
Glóbulos blancos: & $15.8010^{\wedge} 3 / \mathrm{uL}$. \\
Basófilos: & 1,1 por ciento \\
Eosinófilos: & 1,2 por ciento \\
Neutrófilos: & 82,7 por ciento \\
Linfocitos: & 7,5 por ciento \\
Monocitos: & 7,5 por ciento \\
Hematocrito: & 56,4 por ciento \\
Hemoglobina: & 19,4 gramos/ decilitros \\
Glóbulos rojos: & $6,5710^{\wedge} 3 / \mathrm{uL}$. \\
\hline
\end{tabular}


Tabla 3: Prueba Sanguínea

Fuente: Historia Clínica del Paciente - Hospital General IESS Riobamba. 1 de marzo del 2017.

\section{Interpretación de tabla.}

En el siguiente esquema se puede observar que en la biometría hemática existe valores elevados de hemoglobina: $19.4 \mathrm{gr} / \mathrm{dl}$, ya que lo normal es de 11,5 a $16 \mathrm{gr} / \mathrm{dl}$ e igualmente un hematocrito de $56.4 \%$, lo normal es de 38 a 42\%, según la normativa de anemia del ministerio de salud pública, por lo tanto, el paciente muestra una policitemia primaria. La línea blanca se encuentra alterada ya que contamos con leucocitosis de $15.8010^{\wedge} 3 \mathrm{u} / \mathrm{l} \mathrm{y}$ neutrofilia de $82.7 \%$ esto puede ser debido a una infección secundaria de origen desconocido hasta el momento, resto de parámetros se encuentran aparentemente normales.

\begin{tabular}{ll}
\hline Química sanguínea: & \\
\hline Glucosa & $644 \mathrm{mg} / \mathrm{dl}$ \\
Creatinina & $1.5 \mathrm{mg} / \mathrm{dl}$ \\
Ácido úrico & $5.10 \mathrm{mg} / 10$ \\
Nitrógeno ureico & $10 \mathrm{mg} / \mathrm{dl}$ \\
Bilirrubina total & $0.75 \mathrm{mg} / \mathrm{dl}$ \\
Bilirrubina indirecta & $0.52 \mathrm{mg} / \mathrm{dl}$ \\
Bilirrubina directa & $0.23 \mathrm{mg} / \mathrm{dl}$ \\
TGO & 17 \\
TGP & 25 \\
Electrolito Cl & 101 \\
Electrolito k & 5.00 \\
Electrolito NA & 142.0 \\
Hemoglobina glicosilada & 11.9 \\
\hline
\end{tabular}

Tabla 4: Prueba Sanguínea.

Fuente: Historia Clínica del Paciente - Hospital General IESS Riobamba. 1 de marzo del 2017.

\section{Interpretación de Tabla}

En la siguiente tabla, muestra parámetros de química sanguínea, en la que se identifica aumento de la glucosa de $644 \mathrm{mg} / \mathrm{dl}$, ya que los valores normales son de 70 a $110 \mathrm{mg} / \mathrm{dl}$, esto se debe a que existe una descompensación metabólica presuntamente que puede estar desencadenado por un proceso infeccioso, posteriormente revisando los siguientes indicadores encontramos la creatinina dentro de valorares elevados ya que el valor normal es de $1.0 \mathrm{mg} / \mathrm{dl}$, esto puede deberse a la deshidratación que el paciente presenta posterior a danzar, lo cual corrobora los signos en el examen físico, la hemoglobina glicosilada se presenta con $11.9 \%$, siendo el valor normal menos de $5.7 \%$, esto se debe al mal control 
metabólico que el paciente estuvo teniendo por al menos tres meses antes ya sea en su pobre administración de insulina o en la dosificación inexacta que este necesite. Posteriormente el resto de valores se encuentran dentro de rangos normales.

\begin{tabular}{lc}
\hline Elemental y Microscópico de Orina: \\
\hline Bacilos Gram: & Flora \\
Mixta. & 75 \\
Leucocitos & $4+$ \\
Cetonas & $4+$ \\
Glucosa & $0-1 /$ campo \\
Hematíes & $20-50 /$ campo \\
Piocitos & $0-1$ \\
Células epiteliales & +++ \\
Bacterias & Negativo \\
Moco & $0.13 /$ campo \\
Cilindros granulosos & \\
\hline
\end{tabular}

Tabla 5: Examen de Orina

Fuente: Historia Clínica del Paciente - Hospital General IESS Riobamba. 1 de marzo del 2017.

\section{Interpretación de Tabla.}

En la siguiente tabla se identifica una Infección de Vías Urinarias Asintomática, ya que en el estudio de Gram vemos una flora mixta cuando se reporta ello nos indica infección de tracto urinario, corrobora la presencia de bacterias positivas, además de leucocitos y piocitos. El paciente además presenta cuerpos cetónicos que puede estar guiándonos a una complicación aguda de la diabetes mellitus que es la cetoacidosis diabética teniendo en cuenta su patología de base.

Tabla 6: Gasometría arterial

\begin{tabular}{ll}
\hline Gasometría & \\
\hline PH & 7.28 \\
Bicarbonato & $9.2 \mathrm{mmol} / \mathrm{L}$ \\
$\mathrm{PCO} 2$ & $19.7 \mathrm{mmHg}$ \\
$\mathrm{PO} 2$ & $76.0 \mathrm{mmHg}$ \\
$\mathrm{SO} 2 \%$ & $92.1 \%$ \\
Potasio & $4 \mathrm{mmol} / \mathrm{L}$ \\
Cloro & $106.9 \mathrm{mmol} / \mathrm{L}$ \\
Sodio & $139 \mathrm{mmol} / \mathrm{L}$ \\
\hline
\end{tabular}

Fuente: Historia Clínica del Paciente - Hospital General IESS Riobamba. 1 de marzo del 2017. 


\section{Interpretación de Tabla.}

En la siguiente tabla podemos identificar que el paciente se encuentra con acidosis metabólica descompensada, ya que los valores del $\mathrm{PH}$, bicarbonato y presión de $\mathrm{CO} 2$ se encuentran por debajo de su valor normal, esto se da como parte de la complicación aguda de la diabetes mellitus que es la cetoacidosis.

\section{Osmolaridad plasmática del paciente}

$$
\begin{aligned}
& =2(\mathrm{Na})+\frac{\text { Glucosa }}{18}+\frac{\text { BUN }}{2.8} \\
& =323.2 \mathrm{mOsm}
\end{aligned}
$$

\section{Interpretación}

Según los datos de laboratorio del paciente podemos constatar que la Osmolaridad plasmática es de 323.2 mOsm con lo que se llega a pensar en un estado hiperosmolar, causado por un proceso de deshidratación intracelular, es por eso que el paciente presenta la necesidad de beber agua.

\section{DIAGNÓSTICO}

- Diabetes mellitus tipo 2 descompensada

- Cetoacidosis diabética

- Estado hiperosmolar

- Infección de vías urinarias

\section{TRATAMIENTO}

A continuación, se describe el tratamiento tanto farmacológico y no farmacológico, que se le realizó al paciente durante su hospitalización en el Hospital General IESS Riobamba. 
Vol. 3, N².1, p. 7-24, abril - junio, 2019

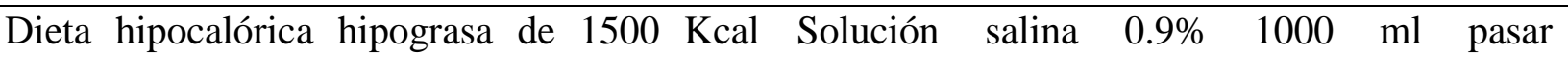
fraccionada en 5 tomas. intravenoso a $100 \mathrm{ml} / \mathrm{h}$.

Control de signos vitales. Tensión arterial, Paracetamol 1 gramo vía oral cada 8 horas. frecuencia respiratoria, saturación de oxígeno.

Control de ingesta y eliminación.

Ceftriaxona 1 gramo intravenoso cada 12 horas.

Control de glicemia capilar.

Complejo B vía oral cada 12 horas.

Exámenes de laboratorio: Biometría hemática, Química sanguínea, Tipificación, Gasometría, Examen Elemental y Microscópico de Orina.

Insulina NPH: 24 UI AM antes del desayuno

14 UI PM antes de la merienda.

Insulina Cristalina por escapes si glucosa es:

$$
\begin{array}{lll}
200 & -250 & 2 \mathrm{UI} \\
251 & -300 & 4 \mathrm{UI} \\
301 & -350 & 6 \mathrm{UI} \\
\text { mayor } 350 & 8 \mathrm{UI}
\end{array}
$$

TABLA 7: Tratamiento

Fuente: Historia Clínica del Paciente - Hospital General IESS Riobamba

\section{EVOLUCIÓN INTRAHOSPITALARIA}

El paciente evoluciona favorablemente de su descompensación metabólica se logra estabilizarlo con el tratamiento indicado por el médico especialista. Su evolución es satisfactoria.

\section{EGRESO DEL PACIENTE.}

Su egreso fue el día 8 de marzo del 2017, en donde al paciente se le envió con medicación para su enfermedad metabólica, se le informa sobre los signos de alarma y se le manda con exámenes de control para nueva valoración con el médico especialista por consulta externa.

\section{SEGUIMIENTO}

A el paciente se le envía un pedido de examen de laboratorio para nueva valoración de glicemia en ayunas y postprandial, para que posteriormente acuda a control en 8 días con resultados por consulta externa.

\section{Conclusiones}


- La Diabetes Mellitus es una patología que no debe sorprender al médico en el momento de su identificación. Para esto, se planteó el análisis de los factores de riesgo, causas y clínica, con el propósito de llegar al diagnóstico antes de estudios complementarios como son los de laboratorio.

- La patología de base del paciente junto con algunos factores de riesgo que presenta la misma como la obesidad, mal manejo terapéutico por parte del paciente y sobrepeso Grado I, han sido consecuencias para que se presente CAD y estado hiperglicémico hiperosmolar no cetósico. Como se evidencia existen datos de múltiples estudios clínicos y casos que indican los principales factores de riesgo.

- Las características clínicas que el paciente presenta es la alteración de la conciencia, polidipsia, náuseas, vómitos y una hiperglicemia severa, los mismos ayudaron a diagnosticar las dos complicaciones de la diabetes que son cetoacidosis diabética y el estado hiperglicemico hiperosmolar no cetósico que de manera combinada se constituye como Ketosis prone.

- Los resultados de los exámenes complementarios muestran datos que reflejan el estado de salud y la condición del paciente.

- La etiopatogenia es aún desconocida; a estos pacientes se les ha descrito alteración en la secreción de insulina por una destrucción no autoinmune de las células beta, el grado de deficiencia insulina es variable y los requerimientos de ésta son fluctuantes.

- En el estudio realizado por Sidana JK, Phillips C, Sinha A, Levy N indica que los principales factores de riesgo para desencadenar las complicaciones de diabetes mellitus son los antecedentes de poseer esta enfermedad, mal manejo por parte del paciente y obesidad entre las más relevantes. En nuestro paciente se cumple con similitud todos estos factores de riesgo por lo que podemos entender porque en nuestro paciente se desencadeno estas complicaciones.(5)

- En cuanto al tratamiento, se inició con insulina subcutáneo dos veces al día y de igual manera con el mismo esquema hasta el próximo control. Con el buen control metabólico se logra a los meses de evolución una mejoría, tanto en la resistencia a la insulina como en el déficit de su secreción, lo que permite suspender el tratamiento insulínico y manejar a los pacientes con metformina o sulfonilureas.

- Según un estudio realizado por Goodstein, G.; Milanesi, A.; y Weinreb,J.E. en una población veterana observaron que más del $50 \%$ de la nueva aparición de diabetes con cetosis es reversible, y el seguimiento temprano después del alta es esencial para guiar el manejo apropiado, incluyendo la posible suspensión de insulina. (11)

- Según la Guía de Práctica Clínica de Diabetes Mellitus tipo 2 del 2017 del Ecuador indica que el tratamiento con insulina se debe realizar bajo tres circunstancias, cuando los niveles de hemoglobina glicosilada supere al 9\%, cuando a pesar de haber administrado antidiabéticos orales por tres meses, no se logra niveles normales de hemoglobina glicosilada y en descompensaciones agudas. (2) En nuestro paciente se dio un tratamiento adecuado logrando aún más la descompensación y un desenlace fatal, con esto nos podemos dar cuenta que como Hospital General IESS Riobamba estamos aptos y preparados para poder revertir este tipo de escenarios.

- Después de la investigación respectiva que se realizó sobre los elementos clínicos y exámenes de laboratorio que nos permitan llegar al diagnóstico de estas complicaciones, puedo comentar que al tratarse de un centro de salud de nivel tipo II 
se pudo realizar exámenes básicos pero decisivos para llegar a determinar las complicaciones que el paciente tuvo al no mantener un buen control metabólico.

- Como sabemos la cetoacidosis y el estado hiperglicemico hiperosmolar son complicaciones agudas de la diabetes a consecuencia de una disminución de la secreción o función de la insulina, normalmente cada una de ellas se da por separado pero en este caso la clínica y los exámenes de laboratorio arrojan criterios para las dos entidades, llegando a la conclusión de que se trata de una entidad mixta que se denomina ketosis prone.

- Es muy importante que una persona con diabetes mellitus se realice los exámenes y controles periódicamente, para identificar complicaciones que pueden presentarse en un tiempo indeterminado y proporcionar el tratamiento oportuno con la medicación indicada.

- El tratamiento recomendado para el paciente, fue el acertado ya que se logró dar una solución urgente y adecuada, estabilizándolo metabólicamente y permitiendo reducir el riesgo de mortalidad.

- El control que el paciente debe realizarse después del tratamiento debe ser meticuloso, mediante la cuantificación de glicemia en ayunas y postprandial la cual debe ser de forma ambulatoria, junto con la medicación y dosificación adecuada la cual no se debe interrumpir.

\section{Referencias Bibliograficas}

1. Zamani-alavijeh F, Araban M, Mohammadi V, Goodarzi F. Development and psychometric evaluation of a new instrument to assess nutritional perceptions and behaviors of diabetic men. Diabetes Metab Syndr Clin Res Rev [Internet]. julio de 2017 [citado 9 de octubre de 2017]; Disponible http://linkinghub.elsevier.com/retrieve/pii/S1871402117301959

2. Medina AMG, others. Guía de Práctica Clínica (GPC) para el diagnóstico, tratamiento y seguimiento de la diabetes mellitus tipo 1 en la población mayor de 15 años. Rev Colomb Endocrinol Diabetes Metab. 2017;3(2):5-21.

3. Censos IN de E y. Diabetes y enfermedades hipertensivas entre las principales causas de muerte en el 2013 [Internet]. Instituto Nacional de Estadística y Censos. [citado 12 de octubre de 2017]. Disponible en: http://www.ecuadorencifras.gob.ec/diabetes-yenfermedades-hipertensivas-entre-las-principales-causas-de-muerte-en-el-2013/

4. Hoyos JD, Jaimes S, Alvear JD, Toloza NA, Vásquez DM, Velandia JN, et al. Factores fisiopatológicos y metas terapéuticas perioperatorias que influyen en procedimientos quirúrgicos reconstructivos mediante colgajos en pacientes con Diabetes Mellitus. Rev MÉDICAS UIS. 2017;30(1):35-43. 
5. Sidana JK, Phillips C, Sinha A, Levy N. Therapeutic challenges in the management of diabetic ketoacidosis. J Intensive Care Soc. noviembre de 2016;17(4):353-5.

6. Farreras, Valent., Rozman, C. Farreras-Rozman Medicina Interna. 17 va Edición. Vol. Volumen 2. España: ELSEVIER; 2012. 1759-1791 p.

7. Ministerio de Salud Publica. GUÍA DE PRACTICA CLINICA DE DIABETES MELLITUS TIPO 2 [Internet]. Primera Edición. Quito: Direccion Nacional de Normatización; 2017. Disponible en: http://www.salud.gob.ec.

8. Goldman, Lee., Ausiello, Dennis. Cecil Tratado de Medicina Interna. 23. a Edición. Vol. Volumen II. España: Elsevier España; 2013.

9. AMERICAN DIABETES ASSOCIATION. STANDARDS OF MEDICAL CARE IN DIABETES 2017. J Clin Appl Res Educ. enero de 2017; Volumen 40(1).

10. Nicolau, Joana., Gimenez, Marga, Miró, Óscar. HIPOGLUCEMIA. JANO. noviembre de 2014; Volumen 10:37-50.

11. Goodstein G, Milanesi A, Weinreb JE. Ketosis-Prone Type 2 Diabetes in a Veteran Population. Diabetes Care. abril de 2014;37(4):e74-5.

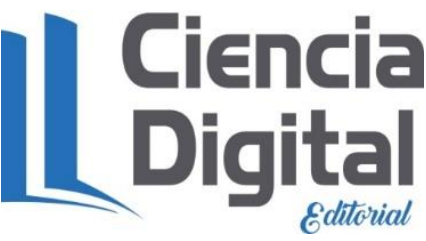




\section{Para citar el artículo indexado.}

Moreno F., Montejo P. \& Polanco A. (2019) Sistema Automático para Cultivos Hidropónicos Domésticos, a través de la Medición de Iones Inorgánicos.

Revista electrónica Ciencia Digital 3(2), 281-291. Recuperado desde: http://cienciadigital.org/revistacienciadigital2/index.php/CienciaDigital/article/view/409/931

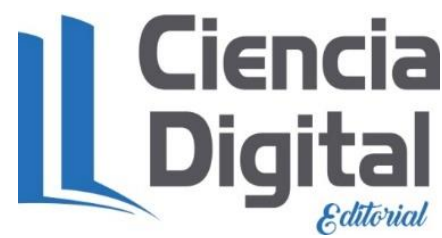

El artículo que se publica es de exclusiva responsabilidad de los autores y no necesariamente reflejan el pensamiento de la Revista Ciencia Digital.

El articulo queda en propiedad de la revista y, por tanto, su publicación parcial y/o total en otro medio tiene que ser autorizado por el director de la Revista Ciencia Digital.
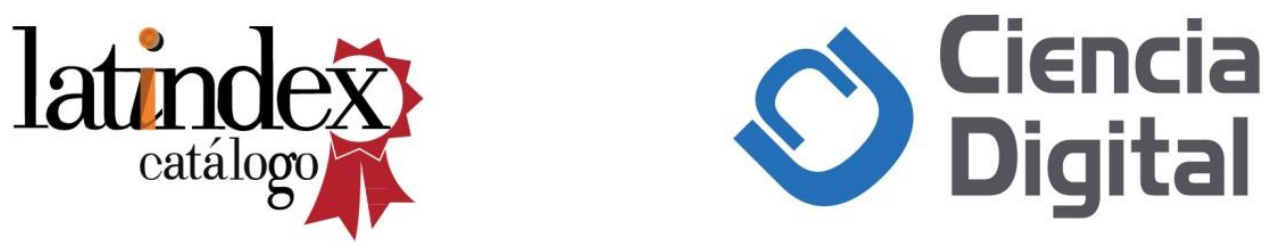